\title{
Comparison of clinical characteristics and performance of pneumonia severity score and CURB-65 among younger adults, elderly and very old subjects
}

\author{
Jung-Hsiang Chen, ${ }^{1,2}$ Shy-Shin Chang, ${ }^{2,3}$ Jason J Liu, ${ }^{4}$ Rai-Chi Chan, ${ }^{5,6}$ \\ Jiunn-Yih Wu, ${ }^{3}$ Wei-Chuan Wang, ${ }^{5,6}$ Si-Huei Lee, ${ }^{5,6,7}$ Chien-Chang Lee ${ }^{4,8}$
}

${ }^{1}$ Department of Family Medicine, Chang Gung Memorial Hospital, Chiayi, Taiwan

${ }^{2}$ Graduate Institute of Clinical Medical Sciences, Chang Gung University, Taoyuan, Taiwan

${ }^{3}$ Department of Family Medicine and Emergency Medicine,

Chang Gung Memorial Hospital, Taoyuan, Taiwan

${ }^{4}$ Department of Epidemiology, Harvard School of Public Health, Boston, USA

${ }^{5}$ Department of Rehabilitation and Physical Medicine, National Yang-Ming University, Taipei, Taiwan

${ }^{6}$ Department of Rehabilitation and Physical Medicine, Taipei Veterans General Hospital, National Yang-Ming University, Taipei, Taiwan

${ }^{7}$ Center for Geriatrics and Gerontology, Taipei Veterans General Hospital, National Yang-Ming University, Taipei, Taiwan

${ }^{8}$ Department of Emergency Medicine, National Taiwan University Hospital, Taipei, Taiwan

\section{Correspondence to}

Si-Huei Lee, Department of Rehabilitation and Physical Medicine, Taipei Veterans General Hospital, No 201, Sec 2, Shipai Road, Taipei 112, Taiwan; sihuei.lee@gmail.com Or

Chien-Chang Lee, Department of Emergency Medicine, National Taiwan University Hospital, No 7, ChungShan South Road, Taipei 100, Taiwan; cclee100@gmail.com

Received 19 October 2009 Accepted 22 July 2010

\begin{abstract}
Background Age-related alterations in the clinical characteristics and performance of severity scoring systems for community-acquired pneumonia (CAP) are unknown.
\end{abstract}

Methods Consecutive patients with CAP presenting to the emergency department were prospectively studied. Patients were classified as younger adults lage 18-64 years), elderly (age 65-84 years) and very old subjects (age $\geq 85$ years). Clinical characteristics, complications, outcomes and validity of the pneumonia severity index (PSI) and CURB-65 categories were compared across these three age categories.

Results Analysis involved 348 (35.3\%) younger adult patients, $438(44.3 \%)$ elderly patients and 201 (20.0\%) very old patients. Compared with younger adults, elderly and very old patients had a higher burden of comorbidities and a higher incidence of CAP-related complications. The 30 -day mortality rate was $5.2 \%$ in younger adults, $7.1 \%$ in elderly patients and $9.5 \%$ in very old patients. The area under the ROC curve (AUCs) for PSI were 0.87 (95\% Cl 0.77 to 0.97$), 0.85$ (95\% Cl 0.803 to 0.897$)$ and $0.69(95 \% \mathrm{Cl} 0.597$ to 0.787$)$ and the AUCs for CURB-65 were 0.80 (95\% $\mathrm{Cl} 0.67$ to 0.93$)$, $0.73(95 \% \mathrm{Cl} 0.65$ to 0.82$)$ and $0.60(95 \% \mathrm{Cl} 0.47$ to 0.73 ) in the younger adult, elderly and very old patients, respectively. A modified PSI or CURB-65 excluding the age variable increased the AUC in most age categories. There was no significant effect of age on 30-day mortality after adjusting for other PSI or CURB-65 variables.

Conclusion Elderly patients with CAP have more atypical clinical manifestations and worse outcomes. The underperformance of the PSI in elderly patients may be due to the inappropriate weight given to the age variable. A modification of the cut-off point for PSI or CURB-65 to define severe pneumonia may improve the score performance in elderly patients.

\section{INTRODUCTION}

Community-acquired pneumonia (CAP) is a common cause of increased morbidity and mortality in older patients. ${ }^{12}$ Elderly patients, with many chronic diseases and diminished physiological reserve, are especially susceptible to CAP, which is often accompanied by serious complications and worse prognosis. ${ }^{3-5}$ Clinically, elderly patients with CAP often have non-specific clinical symptoms or subtle functional decline, making an accurate diagnosis and early antimicrobial treatment difficult and further worsening the prognosis. ${ }^{67}$

Although CAP is a major cause of mortality and morbidity in elderly patients, previous studies on signs or symptoms of CAP in elderly patients have limited sample sizes and lack age-related comparisons or details of another fast-growing age population, the very old patients (age $\geq 85$ years). ${ }^{2-9}$ In addition, although the use of the pneumonia severity index (PSI) or CURB-65 has been widely validated and confirmed, ${ }^{10-18}$ there have been concerns of their validity in the elderly population. ${ }^{19-21}$

We have undertaken an age-related comparative analysis of a cohort of consecutive patients with CAP in a large emergency department of a university hospital. We sought to show the age-related contrasts of coexisting chronic diseases, clinical manifestations and CAP-related complications and outcomes among younger adults (age 18-64 years), elderly patients (age 65-84 years) and very old patients (age $\geq 85$ years). We also sought to investigate how the validity of prognostic scoring systems differs across the three age groups of patients.

\section{METHODS}

\section{Study design and setting}

We conducted a prospective observational study between 1 August 2008 and 28 February 2009 in the emergency department (ED) of a university hospital. The university hospital is a 2000-bed primary and tertiary care medical centre with annual visits of 60000 medical patients.

\section{Selection of participants and data collection}

The study population consisted of adults aged $\geq 18$ years consecutively admitted to the ED with a diagnosis of CAP. The diagnosis of CAP was based on the presence of new infiltrates on the chest $\mathrm{x}$-ray together with a compatible history and/or physical findings. Compatible history is defined as having had fever (tympanic temperature $>38.0^{\circ} \mathrm{C}$ ) or hypothermia (tympanic temperature $<36.0^{\circ} \mathrm{C}$ ), cough with or without sputum production, pleuritic chest pain, dyspnoea or conscious change in the eldery patients. Compatible physical findings included tachypnoea (respiratory rate $>25$ breaths/ $\mathrm{min}$ ), cyanosis, strenuous respiratory pattern and changes in breathing sound such as crackles or 
wheezes on auscultation. Patients diagnosed with nosocomial pneumonia were not eligible for the study. Nosocomial pneumonia was defined as development of pneumonia $>48 \mathrm{~h}$ after admission. For patients with repeated episodes of CAP during the study period, only the first episode was considered for the analysis. For all eligible patients, data on demographic characteristics, pre-existing comorbidities, initial vital signs, routine laboratory test results, admission diagnoses and variables needed to determine the PSI and CURB-65 were prospectively collected. Data on arterial blood gas analysis were only available for those with clinical signs of respiratory distress judged by primary care emergency physicians. Patients lacking arterial blood gas data were assumed to have partial pressure of arterial oxygen $>60 \mathrm{~mm} \mathrm{Hg}$ and arterial $\mathrm{pH}>7.35$.

\section{Definitions}

Three groups of patients were defined by age as younger adults (18-64 years), elderly patients (65-84 years) and very old patients ( $\geq 85$ years). Laboratory data were classified as normal or abnormal with commonly-used ranges. Organ failures assessed at ED admission and during hospitalisation were recorded based on the pre-established definitions characterised as follows: (1) acute renal failure, indicated by serum creatinine level $>3.0 \mathrm{mg} / \mathrm{dl}(>265 \mu \mathrm{mol} / \mathrm{l})$ or, in the case of pre-existing renal dysfunction, a doubling of previous serum creatinine values; (2) acute respiratory distress, indicated by pulse oxygen saturation $<90 \%$ at fraction of inspired oxygen $\left(\mathrm{FiO}_{2}\right) 21 \%$ on ED admission; (3) acute liver function impairment, indicated by serum aspartate aminotransferase level $>70 \mathrm{IU} / 1$ or, in the case of pre-existing chronic liver disease, a doubling of previous values; and (4) shock, indicated by arterial systolic blood pressure $<90 \mathrm{~mm} \mathrm{Hg}$ requiring vasopressors. ${ }^{22-24}$ For parapneumonic effusions, both complicated and uncomplicated ones were included. Comorbidities were recorded according to the weighted score proposed by Charlson et al. ${ }^{25}$ The severity of the illness was quantified using the validated PSI and CURB-65. The PSI developed by Fine et $a l^{10}$ uses 20 clinical variables to define five severity classes; we used classes IV and V to define severe pneumonia. The CURB-65 index uses five core clinical features: new onset confusion, urea $>7 \mathrm{mmol} / 1$, respiratory rate 30 breaths/min, systolic blood pressure $<90 \mathrm{~mm} \mathrm{Hg}$ and/or diastolic blood pressure $60 \mathrm{~mm} \mathrm{Hg}$, and age 65 years. ${ }^{11} \mathrm{We}$ defined severe pneumonia as the presence of three or more criteria. The primary outcome of interest was 30-day mortality due to any cause. The secondary outcomes of interest were hospital admission, ICU admission, mechanical ventilation and length of hospital stay.

\section{Statistical analysis}

Numerical variables were first examined by $q-q$ plot and Shapiro-Wilk test for normality of distribution. If the variable was measured in ordinal scale or had a non-normal distribution, the non-parametric Kruskall-Wallis test or Mann-Whitney U test was used for comparison. For categorical variables, a $\chi^{2}$ test or Fisher exact test was used for univariate comparison. The predictive value of the PSI and CURB-65 categories in the three age groups was assessed in two dimensions of metrics, discrimination and calibration. Discrimination was determined by computing the area under the ROC curve (AUC) with $95 \%$ CI. The best cut-off value was determined by the Youden index to optimise the differentiating ability of the test with equal weight given to sensitivity and specificity. To compare the calibration, the crude number and relative frequencies of observed events in each PSI or CURB-65 risk categories were tabulated. To evaluate the influence of the age variable on the performance of the two scoring systems, we removed age from the two scoring systems and compared the AUC of the modified scores with that of the original scores containing age. To examine whether age has an independent effect on the 30-day mortality after adjusting for the remaining PSI or CURB score variables, we performed a multiple logistic regression analysis. Age was entered into the model as a categorical variable with six equallydistanced categories. Category-specific adjusted odd ratios with reference to the youngest age categories were estimated and the log-odds ratios were plotted against the age categories. A test of linear trend was also performed to examine whether there was a significant linear relationship between age and 30-day mortality. All tests were two-tailed and $p$ values $<0.05$ were

Table 1 Characteristics and underlying comorbidities of the studied groups

\begin{tabular}{|c|c|c|c|c|c|}
\hline & $\begin{array}{l}\text { (A) Younger } \\
\text { adults }(\mathrm{n}=348)\end{array}$ & $\begin{array}{l}\text { (B) Elderly } \\
(n=438)\end{array}$ & $\begin{array}{l}\text { (C) Very old } \\
(\mathrm{n}=201)\end{array}$ & $\begin{array}{l}\text { Significant post } \\
\text { hoc comparisons }\end{array}$ & p Value \\
\hline Male gender (\%) & $218(62.6 \%)$ & $279(63.7 \%)$ & $114(56.7 \%)$ & NS & 0.226 \\
\hline \multicolumn{6}{|l|}{ Comorbidities } \\
\hline Diabetes mellitus & $26(7.5 \%)$ & $110(25.1 \%)$ & $45(22.4 \%)$ & $\begin{array}{l}B \text { vs } A \\
C \text { vs } A\end{array}$ & $<0.001$ \\
\hline Cerebrovascular event & $16(4.6 \%)$ & $64(14.6 \%)$ & $41(20.4 \%)$ & $\begin{array}{l}C \text { vs } A \\
C \text { vs } B \\
B \text { vs } A\end{array}$ & $<0.001$ \\
\hline Dementia & $0(0 \%)$ & $28(6.4 \%)$ & $25(12.4 \%)$ & $\begin{array}{l}C \text { vs } A \\
C \text { vs } B \\
B \text { vs } A\end{array}$ & $<0.001$ \\
\hline Chronic obstructive lung disease & $17(4.9 \%)$ & $87(19.9 \%)$ & $36(17.9 \%)$ & $\begin{array}{l}C \text { vs } A \\
B \text { vs } A\end{array}$ & $<0.001$ \\
\hline Heart failure & $29(8.3 \%)$ & $62(14.2 \%)$ & $38(18.9 \%)$ & $\begin{array}{l}C \text { vs } A \\
B \text { vs } A\end{array}$ & 0.001 \\
\hline Old myocardial infarction & $12(3.4 \%)$ & $14(4.8 \%)$ & $11(3.2 \%)$ & NS & 0.542 \\
\hline Chronic renal insufficiency & $10(2.9 \%)$ & $32(7.3 \%)$ & $12(6.0 \%)$ & $\begin{array}{l}B \text { vs } A \\
C \text { vs } A\end{array}$ & 0.024 \\
\hline End-stage renal disease & $17(4.9 \%)$ & $21(4.8 \%)$ & $4(2.0 \%)$ & NS & 0.203 \\
\hline Liver cirrhosis & $7(2.0 \%)$ & $11(2.7 \%)$ & $4(2.0 \%)$ & NS & 0.792 \\
\hline Non-haematogenous malignancies & $49(14.1 \%)$ & $73(16.7 \%)$ & $29(14.4 \%)$ & NS & 0.563 \\
\hline Haematogenous malignancies & $15(4.3 \%)$ & $12(2.7 \%)$ & $5(2.5 \%)$ & NS & 0.371 \\
\hline Charlson score, median (IOR) & $1(0-3)$ & $2(1-3)$ & $2(1-4)$ & $\begin{array}{l}C \text { vs } A \\
B \text { vs } A\end{array}$ & $<0.001$ \\
\hline
\end{tabular}


Table 2 Clinical and laboratory manifestations of community-acquired pneumonia among three different age groups

\begin{tabular}{|c|c|c|c|c|c|}
\hline & $\begin{array}{l}\text { (A) Younger } \\
\text { adults }(n=348)\end{array}$ & $\begin{array}{l}\text { (B) Elderly } \\
(\mathrm{n}=438)\end{array}$ & $\begin{array}{l}\text { (C) Very old } \\
(\mathrm{n}=201)\end{array}$ & $\begin{array}{l}\text { Significant post } \\
\text { hoc comparisons }\end{array}$ & p Value \\
\hline \multicolumn{6}{|l|}{ Vital signs } \\
\hline Tachycardia & $266(76.4 \%)$ & $298(68.0 \%)$ & $113(56.2 \%)$ & $\begin{array}{l}B \text { vs } A \\
C \text { vs } A\end{array}$ & $<0.001$ \\
\hline Tachypnoea (RR>25) & $14(4.0 \%)$ & $46(10.5 \%)$ & $26(12.9 \%)$ & $\begin{array}{l}B \text { vs } A \\
C \text { vs } A\end{array}$ & 0.004 \\
\hline Fever (tympanic temperature $>38^{\circ} \mathrm{C}$ ) & $121(34.8 \%)$ & $144(32.9 \%)$ & $63(31.3 \%)$ & NS & 0.719 \\
\hline Self-reported history of fever & $190(54.6 \%)$ & $222(50.7 \%)$ & $86(42.8 \%)$ & $\begin{array}{l}C \text { vs } A \\
C \text { vs } B\end{array}$ & 0.017 \\
\hline Hypothermia (tympanic temperature $<36^{\circ} \mathrm{C}$ ) & $35(10.1 \%)$ & $39(8.9 \%)$ & $22(11.0 \%)$ & NS & 0.697 \\
\hline Fall or syncope & $0(0 \%)$ & $14(3.2 \%)$ & $7(3.5 \%)$ & $\begin{array}{l}C \text { vs } A \\
C \text { vs } B\end{array}$ & 0.003 \\
\hline \multicolumn{6}{|l|}{ Laboratory } \\
\hline Anaemia $(\mathrm{Hb}<10 \mathrm{mg} / \mathrm{dl})$ & $40(11.5 \%)$ & $76(17.4 \%)$ & $43(21.4 \%)$ & $\begin{array}{l}B \text { vs } A \\
C \text { vs } A\end{array}$ & 0.006 \\
\hline Leukocytosis (WBC >12000/mm³) & $107(30.8 \%)$ & $172(37.3 \%)$ & $90(44.8 \%)$ & $\begin{array}{l}C \text { vs } A \\
C \text { vs } B\end{array}$ & 0.003 \\
\hline Band for leucocyte $>5 \%$ & $21(6.0 \%)$ & $44(10.1 \%)$ & $17(8.5 \%)$ & NS & 0.13 \\
\hline Thrombocytopenia (platelet $<15 k$ ) & $67(19.3 \%)$ & $99(22.6 \%)$ & $55(27.4 \%)$ & NS & 0.088 \\
\hline Hyponatraemia ( $\mathrm{Na}<130 \mathrm{mEq} / \mathrm{l}$ ) & $35(10.1 \%)$ & $79(18.0 \%)$ & $37(18.4 \%)$ & $\begin{array}{l}C \text { vs } A \\
B \text { vs } A\end{array}$ & 0.003 \\
\hline \multicolumn{6}{|l|}{ Complication } \\
\hline Empyema/ parapneumonic effusion & $26(7.5 \%)$ & $40(9.1 \%)$ & $20(10.0 \%)$ & NS & 0.560 \\
\hline Upper gastrointestinal bleeding & $18(5.2 \%)$ & $38(8.7 \%)$ & $22(11.0 \%)$ & $\begin{array}{l}C \text { vs } A \\
B \text { vs } A\end{array}$ & 0.048 \\
\hline Bacteraemia & $18(5.2 \%)$ & $36(8.2 \%)$ & $10(5.0 \%)$ & NS & 0.141 \\
\hline Adult respiratory distress syndrome & $13(3.7 \%)$ & $13(4.6 \%)$ & $5(2.5 \%)$ & NS & 0.443 \\
\hline \multicolumn{6}{|l|}{ Organ failure } \\
\hline Acute respiratory failure & $29(8.3 \%)$ & $69(14.3 \%)$ & $34(17.7 \%)$ & $\begin{array}{l}C \text { vs } A \\
B \text { vs } A\end{array}$ & 0.003 \\
\hline Altered consciousness & $17(4.9 \%)$ & $30(11.0 \%)$ & $45(13.4 \%)$ & $\begin{array}{l}C \text { vs } A \\
B \text { vs } A\end{array}$ & $<0.001$ \\
\hline Acute renal failure & $15(4.3 \%)$ & $45(10.3 \%)$ & $19(9.5 \%)$ & $\begin{array}{l}B \text { vs } A \\
C \text { vs } A\end{array}$ & 0.006 \\
\hline Acute liver impairment & $36(10.3 \%)$ & $19(4.3 \%)$ & $2(1.0 \%)$ & $\begin{array}{l}A \text { vs } B \\
A \text { vs } C\end{array}$ & $<0.001$ \\
\hline Septic shock & $24(6.9 \%)$ & $49(11.2 \%)$ & $25(12.4 \%)$ & $\begin{array}{l}C \text { vs } A \\
B \text { vs } A\end{array}$ & 0.006 \\
\hline Pneumonia severity index (class) & $2(1-3)$ & $4(3-4)$ & $4(3-5)$ & $\begin{array}{l}C \text { vs } A \\
B \text { vs } A \\
C \text { vs } B\end{array}$ & $<0.001$ \\
\hline CURB-65 & $0(0-1)$ & $2(1-3)$ & $2(2-3)$ & $\begin{array}{l}C \text { vs } A \\
B \text { vs } A \\
C \text { vs } B\end{array}$ & $<0.001$ \\
\hline \multicolumn{6}{|l|}{ Outcome } \\
\hline Mortality & $18(5.2 \%)$ & $31(7.1 \%)$ & $19(9.5 \%)$ & NS & 0.159 \\
\hline Admission to ward & $134(38.5 \%)$ & $259(59.1 \%)$ & $125(62.2 \%)$ & $\begin{array}{l}C \text { vs } A \\
B \text { vs } A\end{array}$ & $<0.001$ \\
\hline Admission to ICU & $33(9.5 \%)$ & $79(18.0 \%)$ & $29(14.4 \%)$ & $\begin{array}{l}C \text { vs } A \\
B \text { vs } A\end{array}$ & 0.003 \\
\hline Mechanical ventilation & $35(10.1 \%)$ & $88(20.1 \%)$ & $32(15.9 \%)$ & $\begin{array}{l}C \text { vs } A \\
B \text { vs } A\end{array}$ & 0.001 \\
\hline Length of hospital stay (median, IQR) & $1(0-10)$ & $8(1-16)$ & $9(2-17)$ & $\begin{array}{l}C \text { vs } A \\
B \text { vs } A\end{array}$ & $<0.001$ \\
\hline
\end{tabular}

considered statistically significant. Data were analysed with SAS Version 9.1.3 (SAS Institute Inc) and STATA Version 10 (STATA Inc).

\section{RESULTS}

\section{Patient population}

During the study period, 1310 episodes of CAP confirmed by $\mathrm{x}$-ray were identified upon admission to the ED, of which 74 were excluded because of missing data, 162 were excluded due to a change to the final discharge diagnosis of pulmonary oedema, malignant effusion or metastatic cancer, 50 were excluded because of repeated episodes and 37 were excluded due to do-not-attempt-resuscitation order or terminal illness with palliative care. Ultimately, a total of 987 patients entered the analysis (348 younger adult patients, 294 elderly patients and 345 very old patients).

Patient characteristics and comorbidity

The characteristics of the studied patients are summarised in table 1 . The mean age of the study population was 
Table 3 Comparative validation of CURB-65 and pneumonia severity index (PSI) in three age groups of patients

\begin{tabular}{|c|c|c|c|c|c|}
\hline & Score performance indicators & Whole cohort $(\mathrm{n}=987)$ & Younger adults $(n=348)$ & Elderly $(n=438)$ & Very old $(\mathrm{n}=201)$ \\
\hline \multirow[t]{3}{*}{ PSI } & Area under ROC curve $(95 \% \mathrm{Cl})$ & 0.83 (0.78 to 0.87 ) & 0.87 (0.77 to 0.97 ) & $0.85(0.80$ to 0.90$)$ & 0.69 (0.60 to 0.79$)$ \\
\hline & $\begin{array}{l}\text { Sensitivity, specificity under } \\
\text { recommended cut-off point }\end{array}$ & IV $(95.6 \%, 51.3 \%)$ & IV $(88.9 \%, 80.6 \%)$ & IV $(100 \%, 38.3 \%)$ & IV $(100 \%, 19.8 \%)$ \\
\hline & $\begin{array}{l}\text { PPV, NPV under recommended cut-off } \\
\text { point }\end{array}$ & IV $(21.7 \%, 97.5 \%)$ & IV $(33.3 \%, 97.5 \%)$ & IV $(23.9 \%, 98.5 \%)$ & IV (14.9\%, 94.7\%) \\
\hline \multirow[t]{4}{*}{ CURB-65 } & Area under ROC curve $(95 \% \mathrm{CI})$ & 0.73 (0.67 to 0.79 ) & 0.80 (0.67 to 0.93 ) & 0.73 (0.65 to 0.82 ) & 0.60 (0.48 to 0.73 ) \\
\hline & $\begin{array}{l}\text { Sensitivity, specificity under } \\
\text { recommended cut-off point }\end{array}$ & $3(81.9 \%, 48.5 \%)$ & $3(22.2 \%, 99.7 \%)$ & $3(64.5 \%, 74.7 \%)$ & $3(47.4 \%, 65.9 \%)$ \\
\hline & $\begin{array}{l}\text { Sensitivity, specificity under adjusted } \\
\text { cut-off point }\end{array}$ & $2(83.8 \%, 52.3 \%)$ & $2(61.1 \%, 95.8 \%)$ & $3(64.5 \%, 74.7 \%)$ & $3(47.4 \%, 65.9 \%)$ \\
\hline & $\begin{array}{l}\text { PPV, NPV under recommended cut-off } \\
\text { point }\end{array}$ & $3(16.6 \%, 95.6 \%)$ & $3(80.0 \%, 95.9 \%)$ & $3(16.3 \%, 96.5 \%)$ & $3(12.7 \%, 92.3 \%)$ \\
\hline
\end{tabular}

NPV, negative predictive value; PPV, positive predictive value.

$68.0 \pm 19.3$ years and the male to female ratio was 0.62 . The elderly and very old patients had more diabetes mellitus, stroke, dementia, chronic lung and renal disease so they had higher mean Charlson scores. Compared with the elderly or younger adult patients, the very old patients had a higher prevalence of stroke and dementia. We did not find malignancies over-represented in the elderly patients in our sample, but we did find a relatively higher proportion of patients with head and neck or oesophageal cancer in younger adults. These types of cancer are more likely to develop in younger adults and predispose them to recurrent aspiration pneumonia due to swallowing dysfunction.

\section{Clinical and laboratory manifestations}

The clinical manifestations and laboratory results are summarised in table 2. Compared with younger adult patients, the elderly and very old patients had significantly less tachycardia and a self-reported history of fever and significantly more tachypnoea and syncope or falls. We did not find significantly more elderly or very old patients to have lower measured body temperature on ED admission. For laboratory findings, the elderly and very old patients had significantly higher prevalence of anaemia, leukocytosis and hyponatraemia than younger adult patients. The laboratory findings of the very old patients are generally comparable to the elderly patients.

\section{Complications, organ failure and outcome}

CAP-related complications, organ failures and outcomes are also summarised in table 2. For complications, we did not find the elderly or very old patients to be more likely to develop empyema, parapneumonic pleural effusions, bacteraemia or adult respiratory distress syndrome. However, we found that the elderly and very old patients were more prone to develop acute gastrointestinal bleeding and organ dysfunction. A significantly higher proportion of younger adult patients also developed acute liver impairment, which is a rarely reported finding. For major outcomes, the study cohort had a 30-day all-cause mortality of $6.9 \%$, an ICU admission probability of $14.3 \%$ and a mean length of hospital stay of $10.6 \pm 16.3$ days. Although the trend of higher mortality with increasing age is significant $\left(p_{\text {trend }}=0.019\right)$, the sample size is not sufficiently powered to detect the mortality difference between the three groups of patients. Other than mortality, the elderly and very old patients had a significantly higher probability of ward and ICU admissions, a higher probability of ICU admission, higher demand for mechanical ventilation and longer length of stay in hospital.

\section{Validity of PSI and CURB-65}

Table 3 shows the predictive accuracy of the PSI and CURB-65 for 30-day mortality. Both PSI and CURB-65 showed a trend of decreasing AUC in relation to higher age categories (figure 2). The best discriminative power of PSI and CURB-65 was achieved by different cut-off points in different age groups. Younger adult patients require a lower threshold value to distinguish severe from non-severe pneumonia than elderly or very old patients. On average, the PSI had high sensitivity and a high negative predictive value (NPV) while CURB-65 had low sensitivity but also a high NPV in predicting 30-day mortality. For both PSI and CURB-65, the NPV remained stable across age groups based on the recommended threshold value, while the positive predictive value (PPV) declined dramatically with advancing age categories. Table 4 summarises the crude number and proportion of non-survivors in each risk prediction category stratified by age groups.

\section{Influence of age variable}

As the PSI gives linear weight to the age variable, the severity scores of elderly and very old patients were indiscriminately upgraded, resulting in a low specificity in predicting 30-day mortality. To evaluate whether age was still linearly associated with the risk of 30-day mortality after adjusting for PSI and CURB variables, we performed logistic regression analysis and plotted log-OR versus 10-year age categories (figure 1).

Table 4 30-day mortality by different severity classes of CURB-65 and pneumonia severity index (PSI) in three age groups of patients

\begin{tabular}{llll}
\hline PSI classification & Class I/II & Class III & Class IV $\mathbf{N}$ \\
\hline Whole cohort & $0.4 \%(1 / 266)$ & $0.5 \%(1 / 194)$ & $12.5 \%(66 / 527)$ \\
Age $18-64$ & $0.4 \%(1 / 223)$ & $2.2 \%(1 / 45)$ & $20.0 \%(16 / 80)$ \\
Age 65-84 & $0 \%(0 / 43)$ & $0 \%(0 / 113)$ & $11.0 \%(31 / 282)$ \\
Age $\geq 85$ & - & $0 \%(0 / 36)$ & $11.5 \%(19 / 165)$ \\
CURB-65 category & $\mathbf{0 - 1}$ & $\mathbf{2}$ & $\mathbf{3}-\mathbf{5}$ \\
Whole cohort & $2.4 \%(12 / 493)$ & $7.8 \%(23 / 295)$ & $16.6 \%(33 / 199)$ \\
Age 18-64 & $2.5 \%(8 / 324)$ & $31.6 \%(6 / 19)$ & $80.0 \%(4 / 5)$ \\
Age 65-84 & $1.6 \%(2 / 127)$ & $4.8 \%(9 / 188)$ & $16.3 \%(20 / 123)$ \\
Age $\geq 85$ & $4.8 \%(2 / 42)$ & $9.6 \%(8 / 88)$ & $23.1 \%(9 / 71)$ \\
\hline
\end{tabular}


Figure $1(\mathrm{~A}-\mathrm{C})$ Crude or adjusted log-odds ratio of 30-day mortality for different age groups. After adjustment with pneumonia severity index (PSI) or confusion, urea, respiratory rate, blood pressure (CURB) variables, the effect of age on 30-day mortality was not significant.
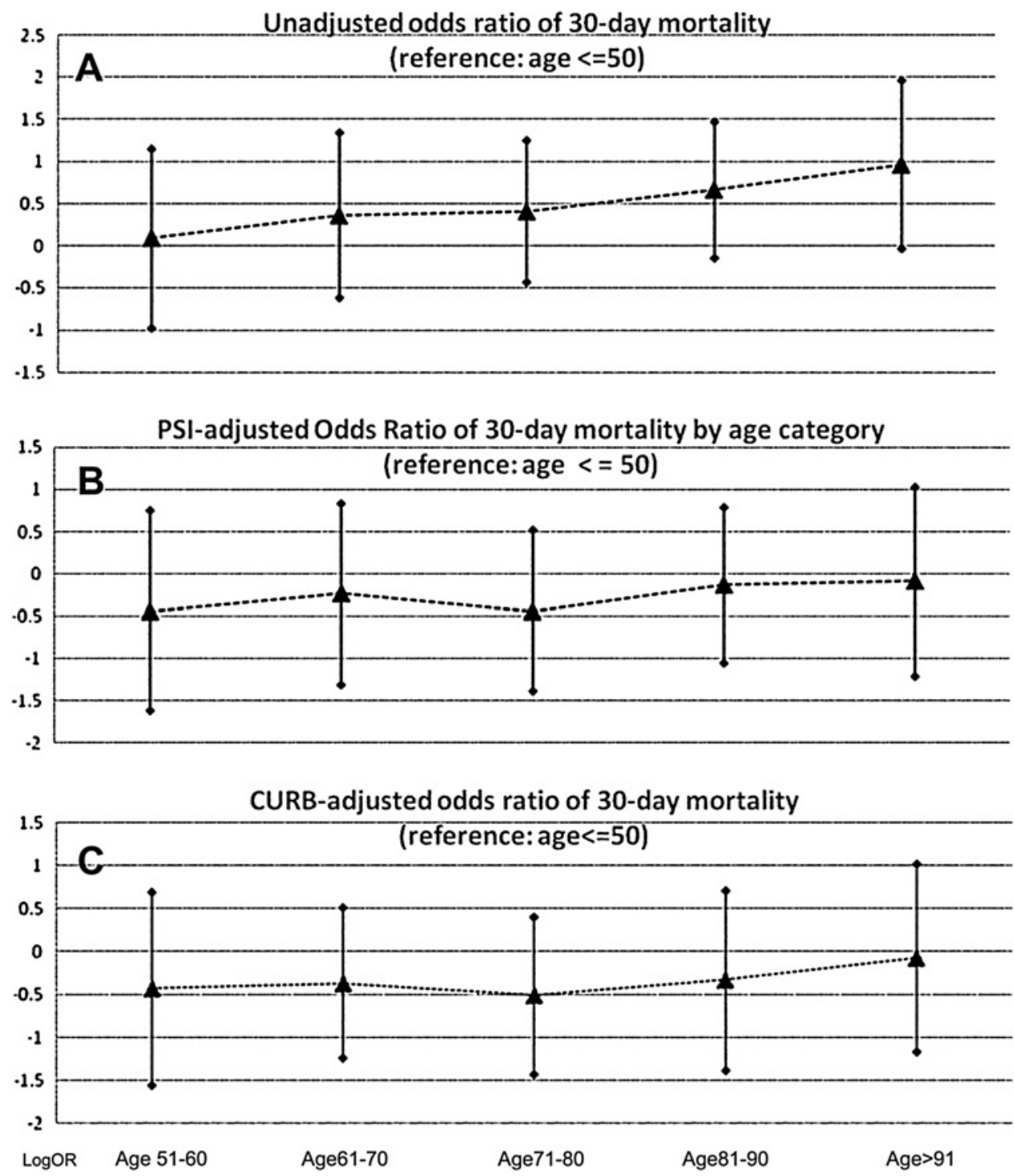

Compared with the reference population (age $\leq 50$ years), the crude risk of 30-day mortality increased linearly with advancing 10 -year age categories $\left(p_{\text {trend }}=0.027\right.$, figure $1 \mathrm{~A}$ ). After adjusting for PSI or CURB variables, there were no significant residual effects of age on 30 -day mortality $\left(p_{\text {trend }}=0.907\right.$ for PSI, figure $1 \mathrm{~B}$; $\mathrm{p}_{\text {trend }}=0.357$, figure $1 \mathrm{C}$ ). We also performed ROC analysis for modified PSI and CURB that excluded the age variable. Compared with the original scores, the modified PSI or CURB scores had better AUC in most age categories, except the slightly decreased AUC of the modified CURB-65 score in the very old patients (figure $2 \mathrm{~A}-\mathrm{D}$ ). Due to sample size limitation, none of these comparisons was statistically significant.

\section{DISCUSSION}

The geriatric population ( $\geq 65$ years) represents the fastest growing segment of the general population, with the very old segment ( $\geq 85$ years) expanding most rapidly. ${ }^{26}$ Despite this fact, there have rarely been studies comparing the outcomes or clinical characteristics of CAP between younger adults and elderly subjects. Our study shows that elderly and very old patients have significantly different clinical presentations of CAP compared with younger adult patients. Both elderly and very old patients had a higher burden of chronic diseases, higher prevalence of anaemia, leukocytosis and hyponatraemia, were more likely to present with atypical clinical manifestations and were more prone to develop organ failure or gastrointestinal bleeding. The very old patients have an even higher propensity to develop organ failure, longer hospital stay and worse outcomes than elderly patients. ${ }^{3-9}$

It has been shown that the established scoring systems for CAP severity may not perform well in elderly patients because their atypical clinical presentations may make the cut-off values for the scoring system unreliable. ${ }^{19} 20$ Our results corroborate this finding by showing a decreasing discriminative power (measured by AUC) of PSI and CURB-65 with advancing age categories. ${ }^{19} 20 \quad 27-29$ An obvious reason for the underperformance of the PSI in elderly patients is the undue weights given to the age variable in the PSI score. As shown in figure 1, there were minimal (if any) residual effects of age on 30-day mortality after adjusting for PSI component variables other than age. Moreover, excluding the age variable from PSI or CURB-65 increased the AUC in most age categories. To improve the performance of PSI in elderly and very old patients, it is imperative to develop a non-linear weight for the age variable in PSI. Before such study is available, we suggest a modification of PSI cut-off points. As shown in our study, a higher PSI cut-off of 105 points (compared with the original 91 points) could achieve a sensitivity of $100 \%$ and a specificity of $55.7 \%$ in the elderly 
Figure $2 \quad(A-D)$ ROC curves of pneumonia severity index (PSI) with/ without age variable and confusion, urea, respiratory rate, blood pressure (CURB)-65/CURB for 30-day mortality in different age populations. Compared with the original scores, the modified scores generally had a larger area under the ROC curve, except the CURB score in the very old patients.
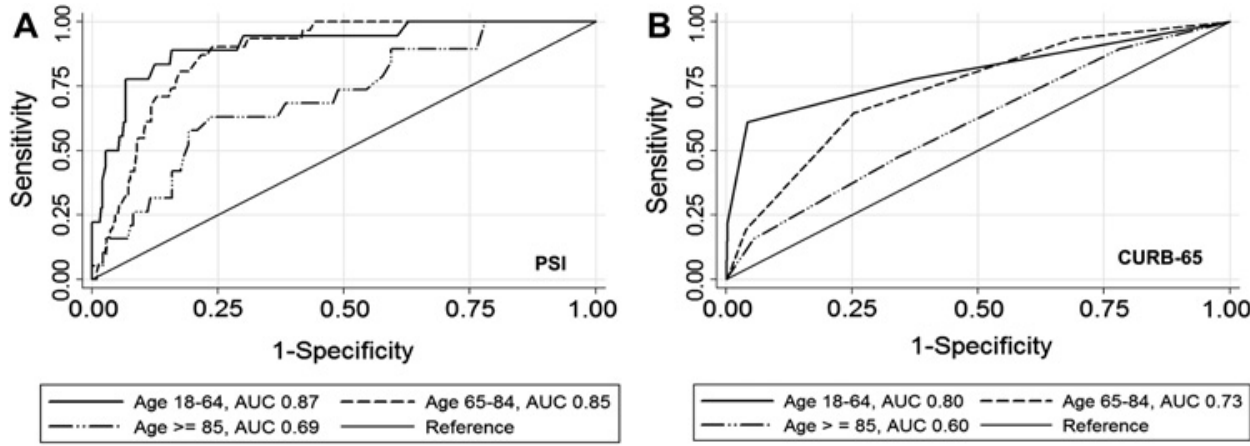
-..- Age $>=85$, AUC $0.60-$ Reference

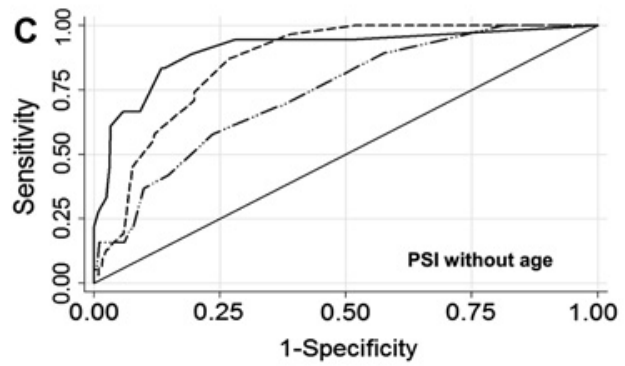

\begin{tabular}{l} 
- Age 18-64, AUC $0.91----$ Age 65-84, AUC 0.86 \\
-..- Age $>=85$, AUC $0.74 \longrightarrow$ Reference \\
\hline
\end{tabular} population, and a cut-off of 108 points could achieve a sensitivity of $90 \%$ and a specificity of $40 \%$ in the very old population.

Although ROC analysis is a popular method of evaluating score performance, judging the performance of a scoring system solely by AUC may lead to misinterpretation in real clinical settings. The AUC is not the probability that individuals are classified correctly, but mainly describes how well cases are ranked higher than non-cases for a given risk classification rule. Cook recently showed that differences between two individuals with very low risk (eg, $1.0 \%$ vs $1.1 \%$ ) have the same effect on the AUC as two individuals with higher risk and a greater risk difference (eg, $5 \%$ vs $20 \%$ ). ${ }^{30}$ In a hypothetical extreme situation, the AUC may remain high even if most non-survivors are misclassified to the low-risk categories as long as the nonsurvivors are ranked higher than survivors within those low-risk categories. The scenario is demonstrated in our study where the younger adult patient group had better AUC for CURB-65 (0.80) than the elderly $(0.73)$ or very old patients $(0.60)$, but 14 out of $18(77.8 \%)$ non-survivors in the younger adult group were misclassified as low risk compared with 11 of 31 (35.4\%) in the elderly group and 10 of $19(52.6 \%)$ in the very old group. We therefore discourage the use of the traditional cut-off point of CURB-65 on the low-risk younger population despite its better AUC. Under the recommended cut-off point, the severity of the younger low-risk patients was probably underestimated.

Past efforts to improve the performance of risk prediction in the elderly population have mainly focused on the development of new scores or the modification of PSI or CURB-65 variables. Pilotto et al stated that the multidimensional prognostic index (MPI) composed of social, educational, functional and medical variables performed better than the PSI in predicting death in elderly patients with CAP. ${ }^{31}$ Naito et al showed that a modified PSI with addition of performance, anorexia and hypercapnia variables may greatly enhance the sensitivity of the original PSI at the cost of dramatically lower specificity. ${ }^{28}$ Mint et al showed that the systolic blood pressure, oxygenation, age and respiratory rate score (SOAR) was comparable but not superior to PSI or CURB-65 in elderly patients. ${ }^{29}$ We agree with these authors that some prognostic information, such as bilateral lobar infiltrates on the x-ray or underlying functional status, are not fully captured by PSI variables but we do not think the addition of one or two variables will greatly improve the performance. ${ }^{928}$ Again, we think the main problem of the poor performance of PSI or CURB-65 in elderly patients lies in the inappropriate weight of the age variable or the inappropriate threshold value to define severe disease.

Compared with previous studies, our study has the strengths of having a prospective design, comparison groups across different age strata, relatively large sample sizes in the elderly and very old populations and inclusion of both inpatients and outpatients. Owing to the inclusion of outpatients, the overall severity of CAP in this study cohort was reduced. The thresholds found in this study may not apply to other patient populations with very different demographic backgrounds. One of the limitations of this study is that multiple comparisons were made in the univariate analysis so we cannot exclude the possibility of false positive findings. We chose not to use the Bonferroni correction because the clinical characteristics were highly correlated and the independency assumption of the Bonferroni correction was violated.

In conclusion, we have shown that the clinical manifestations and outcomes differ in three age groups of patients with CAP. The discriminative capability of PSI or CURB-65 in predicting 30-day mortality for CAP decreased with advancing age. Using the originally recommended cut-off points for PSI or CURB-65 may underestimate the severity in the younger adult population and overestimate the severity in the elderly and very old populations. Future large prospective studies are required to determine the best weight for the age variable in the PSI score or the best age-adjusted threshold value to define severe pneumonia.

Funding This work is supported by grant NSC 97-2321-B-002-014 from the National Science Council of Taiwan. 


\section{Competing interests None.}

Patient consent Obtained

Ethics approval This study was conducted with the approval of the Institutional Review Board, National Taiwan University Hospital.

Provenance and peer review Not commissioned; externally peer reviewed.

\section{REFERENCES}

1. Heron MP, Smith BL. Deaths: leading causes for 2003. Nat/ Vital Stat Rep 2007;55:1-93

2. Jackson ML, Neuzil KM, Thompson WW, et al. The burden of community acquired pneumonia in seniors: results of a population-based study. Clin Infect Dis 2004;39:1642-50.

3. Janssens JP, Gauthey L, Herrmann F, et al. Community-acquired pneumonia in older patients. J Am Geriatr Soc 1996;44:539-44.

4. Lieberman D, Lieberman D, Schlaeffer F, et al. Community-acquired pneumonia in old age: a prospective study of 91 patients admitted from home. Age Ageing 1997:26:69-75.

5. Koivula I, Stén M, Mäkelä PH. Prognosis after community-acquired pneumonia in the elderly: a population-based 12-year follow-up study. Arch Intern Med 1999:159:1550-5.

6. Schneider EL. Infectious diseases in the elderly. Ann Intern Med 1983:98:395-400.

7. Lee CC, Chen SY, Chang IJ, et al. Comparison of clinical manifestations and outcome of community-acquired bloodstream infections among the oldest old, elderly, and adult patients. Medicine (Baltimore) 2007;86:138-44.

8. Jackson ML, Nelson JC, Jackson LA. Risk factors for community-acquired pneumonia in immunocompetent seniors. J Am Geriatr Soc 2009;57:882-8.

9. Mody L, Sun R, Bradley SF. Assessment of pneumonia in older adults: effect of functional status. J Am Geriatr Soc 2006;54:1062-7.

10. Fine MJ, Auble TE, Yealy DM, et al. A prediction rule to identify low-risk patients with community-acquired pneumonia. N Engl J Med 1997;33:243-50

11. Lim WS, van der Eerden MM, Laing $\mathrm{R}$, et al. Defining community acquired pneumonia severity on presentation to hospital: an international derivation and validation study. Thorax 2003;58:377-82.

12. Ewig S, de Roux A, Bauer $\mathrm{T}$, et al. Validation of predictive rules and indices of severity for community acquired pneumonia. Thorax 2004;59:421-7.

13. Aujesky D, Auble TE, Yealy DM, et al. Prospective comparison of three validated prediction rules for prognosis in community-acquired pneumonia. Am J Med 2005;118:384-92.

14. Buising KL, Thursky KA, Black JF, et al. A prospective comparison of severity scores for identifying patients with severe community acquired pneumonia: reconsidering what is meant by severe pneumonia. Thorax 2006;61:419-24.
15. Capelastegui A, España PP, Quintana JM, et al. Validation of a predictive rule for the management of community-acquired pneumonia. Eur Respir J 2006;27:151-7.

16. Man SY, Lee N, Ip M, et al. Prospective comparison of three predictive rules for assessing severity of community-acquired pneumonia in Hong Kong. Thorax 2007;62:348-53

17. Barlow G, Nathwani D, Davey P. The CURB65 pneumonia severity score outperforms generic sepsis and early warning scores in predicting mortality in community-acquired pneumonia. Thorax 2007;62:253-9.

18. Valencia M, Badia JR, Cavalcanti $\mathbf{M}$, et al. Pneumonia severity index class v patients with community-acquired pneumonia: characteristics, outcomes, and value of severity scores. Chest 2007;132:515-22.

19. Mody L, Sun R, Bradley S. Community-acquired pneumonia in older veterans: does the pneumonia prognosis index help? J Am Geriatr Soc 2002;50:434-8.

20. Parsonage $\mathbf{M}$, Nathwani D, Davey $P$, et al. Evaluation of the performance of CURB 65 with increasing age. Clin Microbiol Infect 2009;15:858-64.

21. Niederman MS. Making sense of scoring systems in community acquired pneumonia. Respirology 2009:14:327-35.

22. American College of Chest Physicians/Society of Critical Care Medicine Consensus Conference. Definitions for sepsis and organ failure and guidelines for the use of innovative therapies in sepsis. Crit Care Med 1992;20:864-74.

23. Pittet D, Harbarth S, Suter PM, et al. Impact of immunomodulating therapy on morbidity in patients with severe sepsis. Am J Respir Crit Care Med 1999:160:852-7.

24. Hebert PC, Drummond AJ, Singer J, et al. A simple multiple system organ failure scoring system predicts mortality of patients who have sepsis syndrome. Chest 1993:104:230-5.

25. Charlson ME, Pompei P, Ales KL, et al. A new method of classifying prognostic comorbidity in longitudinal studies: development and validation. J Chronic Dis 1987; 40:373-83

26. Schneider EL. Aging in the third millennium. Science 1999;283:796-7.

27. Higuchi T, Ota $\mathrm{K}$, Tanabe $\mathrm{Y}$, et al. [Severity classification and prognosis in hospitalized elderly patients with community-acquired pneumonia]. Nippon Ronen Igakkai Zasshi 2007:44:483-9.

28. Naito T, Suda T, Yasuda $\mathrm{K}$, et al. A validation and potential modification of the pneumonia severity index in elderly patients with community-acquired pneumonia. J Am Geriatr Soc 2006;54:1212-19.

29. Mint PK, Kamath AV, Vowler SL; British Thoracic Society: Severity assessment criteria recommended by the British Thoracic Society (BTS) for community-acquired pneumonia (CAP) and older patients. Should SOAR (systolic blood pressure, oxygenation, age and respiratory rate) criteria be used in older people? A compilation study of two prospective cohorts. Age Ageing 2006;35:286-91.

30. Cook NR. Use and misuse of the receiver operating characteristic curve in risk prediction. Circulation 2007;115:928-35.

31. Pilotto A, Addante F, Ferrucci $L$, et al. The multidimensional prognostic index predicts short- and long-term mortality in hospitalized geriatric patients with pneumonia. J Gerontol A Biol Sci Med Sci 2009;64:880-7. 